\title{
Cadena de valor del cocotero de la Costa Grande de Guerrero, México
}

\author{
Lizelia Castro-Gil ${ }^{1}$, José Alberto Solis-Navarrete 2,3, Priscila Ortega-Gómez ${ }^{4}$, María \\ Xochitl Astudillo-Miller ${ }^{1}$
}

1 Unidad de Estudios de Posgrado e Investigación de la Universidad Autónoma de Guerrero; ${ }^{2}$ Centro de Investigaciones en Geografía Ambiental de la Universidad Nacional Autónoma de México;

3isolia@ciga.unam.mx; ${ }^{4}$ Facultad de Contaduría y Ciencias Administrativas, Universidad Michoacana de San Nicolás de Hidalgo

Castro-Gil Lizelia, José Alberto Solis-Navarrete, Priscila Ortega-Gómez, María Xochitl Astudillo-Miller (2020). Cadena de valor del cocotero de la Costa Grande de Guerrero, México. Rev. Fac. Agron. Vol 119 (2): 1-9. https://doi.org/10.24215/16699513e058

El cultivo de coco en Guerrero, México genera 17,370 empleos de los cuales 8,463 se encuetran en la región Costa Grande donde la copra es el principal producto, caracterizado por la baja adición de valor. La investigación tiene el objetivo de analizar la cadena de valor del sector cocotero en la región a través de fuentes documentales, observación y aplicación de entrevistas semiestructuradas. A pesar de las brechas en la cadena de valor del cocotero, existen oportunidades para la adopción de estrategias a través de mecanismos que beneficien al sector en general, permitiendo la integración de los productores primarios.

Palabras clave: cadena de valor; cocotero; competitividad; Costa Grande de Guerrero

Castro-Gil Lizelia, José Alberto Solis-Navarrete, Priscila Ortega-Gómez, María Xochitl Astudillo-Miller (2020). Coconut value chain of the Costa Grande of Guerrero, Mexico. Rev. Fac. Agron. Vol 119 (2): 1-9. https://doi.org/10.24215/16699513e058

Coconut cultivation in Guerrero, Mexico generates 17,370 jobs, of which 8,463 are in the Costa Grande region where copra is the main product, characterized by low-value addition. The research aims to analyze the value chain of the coconut in the region through documentary sources, observation, and semi-structured interviews. Despite the gaps in the coconut palm value chain, there are opportunities for the adoption of strategies through mechanisms that benefit the sector in general, allowing the integration of primary producers.

Key words: value chain; coconut tree; competitiveness; Gosta Grande of Guerrero

https://revistas.unlp.edu.ar/revagro

Recibido: 04/12/2019

Aceptado: $18 / 02 / 2020$

Disponible on line: 07/12/2020

ISSN 0041-8676 - ISSN (on line) 1669-9513, Facultad de Ciencias Agrarias y Forestales, UNLP, Argentina. 


\section{INTRODUCCIÓN}

De acuerdo con la Organización de las Naciones Unidas para la Alimentación y la Agricultura [FAO], (2017), el continente asiático ha liderado históricamente la oferta mundial de coco. México se encuentra en la séptima posición en la lista de los diez principales países productores a nivel internacional; sin embargo, hay una importante diferencia en la magnitud del volumen de producción en comparación con los países líderes (Indonesia, Filipinas e India), que poseen la mayor parte de la producción con el $81.71 \%$. En América Latina, Brasil concentra el mayor volumen de toneladas producidas $(2,893,385.27)$ por lo que se considera el principal competidor de México. De acuerdo con el Servicio de Información Agroalimentaria y Pesquera (SIAP, 2018), en México la producción de coco se concentra en nueve estados, para el año 2016 se alcanzó un total de 222,018.07 toneladas (t). La mayor superficie sembrada se localiza en la zona del pacífico, en los estados de Guerrero, Colima, Oaxaca, Michoacán, Jalisco y Chiapas, con 113,619.5 hectáreas (ha) (89\%); estas entidades concentran el $95 \%$ de la producción de total.

En este contexto, la participación del estado de Guerrero tiene gran relevancia, ya que representa el $80.6 \%$ de la producción con $179,769.99$ t, posicionándose como el principal productor a nivel nacional, con un rendimiento de $2.15 \mathrm{t} \mathrm{ha-}^{-1}$ siendo superior en comparación con las demás entidades dedicadas a esta actividad, con rendimiento promedio de 1.79 t ha- ${ }^{-1}$. En Guerrero, la palma de coco se cultiva en 22 de sus 81 municipios, destaca por su superficie sembrada y producción de Tecpan de Galeana con 14,961 ha y 12,352.38 t, respectivamente (SIAP, 2018).

Dicho municipio pertenece a la región Costa Grande, en la cual se encuentran ubicadas quince empresas que procesan los diferentes derivados del coco, de la cuales ocho elaboran productos destinados a la industria agroalimentaria y las restantes orientadas a la agroindustria. A pesar de que son 15 las organizaciones más representativas y a su vez encargadas de procesar parte de la producción de coco para adicionar valor agregado, no se encuentran identificadas las actividades más relevantes de cada una de estas, sus problemáticas, necesidades y el progreso que han tenido dentro del sector cocotero.

Por otro lado, el Consejo Estatal del Cocotero Organismo Público Descentralizado (CECOCO O.P.D., 2016), se crea en 2001 por medio del Decreto Núm. 308, como un organismo para impulsar a través de la cohesión de esfuerzos, acciones y recursos de los tres niveles de gobierno y cada uno de los integrantes de la cadena productiva en las diferentes actividades que desempeñan los agentes que conforman al sector. Por lo cual, brinda atención institucionalizada, soporte público de acuerdo con Acosta et al. (2015) y consultoría a las empresas que conforman el sector para la elaboración de proyectos y en la gestión de recursos. Sin embargo, estas acciones deben ir acompañadas de mecanismos que permitan desarrollar el potencial del sector a través del aprovechamiento de los recursos tecnológicos disponibles en la región y su articulación con otros actores (Malerba, 2002), y lograr una demanda sostenible de la nuez de coco. Si bien la creación de empresas que agreguen valor a los diferentes derivados es necesaria e importante, en primera instancia, se debe dotar de capacidades a aquellas existentes, para mejorar los procesos de producción y comercialización de los distintos productos, en donde es importante la participación de las Instituciones de Educación Superior (IES) públicas para el desarrollo $s$ a través de sus aportes a la ciencia, al tratarse de instituciones cuyo interés académico está fuera de compromisos de índole comercial, les concierne tener en cuenta una percepción más amplia y con mayor alcance acerca de las necesidades de un territorio (Arredondo, 1990).

El objetivo de la presente investigación es identificar y analizar la cadena de valor de las empresas más representativas que conforman el sector cocotero en la región Costa Grande del estado de Guerrero. El modelo aplicado por Porter (2015) desde en enfoque para el sector (Sagheer y Yadav, 2009; Kumar y Kapoor, 2010), al clasificar las actividades que realizan las organizaciones en: actividades primarias y actividades secundarias. Cada una de estas categorías, a su vez comprenden un conjunto de acciones, las primeras relacionadas con la elaboración de un producto, su venta y transferencia al cliente y en la asistencia brindada posteriormente, estas actividades se respaldan por las actividades de apoyo. Las actividades primarias se conforman por cinco categorías genéricas, que a su vez se dividen en subactividades definidas que varían dependiendo la industria en cuestión y de la estrategia que implemente la empresa.

- Logística de entrada: incorpora las actividades relacionadas con la recepción, almacenamiento y distribución de los insumos del producto.

- Operaciones: se incluyen todas las actividades necesarias para la transformación de los insumos en el producto final.

- Logística de salida: reúne las actividades que implican la obtención, almacenaje y distribución del producto entre los clientes.

- Mercadotecnia y ventas: se trata de las actividades que proporcionan los recursos que permite al cliente la compra del producto y que la empresa lo incite a ello.

- Servicio: agrupa las actividades que brindan un servicio para mejorar o conservar el valor del producto.

Por otro lado, las actividades de apoyo se dividen en cuatro categorías, cada una de las cuales se subdividen en las siguientes actividades:

- Adquisición: comprende la compra de insumos requeridos en la cadena de valor, que se realiza en los diferentes departamentos de la empresa, e incluyen la materia prima, suministros y otros componentes consumibles, maquinaria, equipo de oficina, edificios, etc

- Desarrollo tecnológico: actividades mediante las cuales la tecnología empleada en los procesos, los procedimientos prácticos, los métodos agregan valor a través de la mejora en las características e imagen el producto o del proceso de producción y comercialización.

- Administración de recursos humanos: esta categoría concentra las actividades relacionadas con el reclutamiento, la contratación, la capacitación, el desarrollo y compensación de todo el personal. 
- Infraestructura organizacional: se trata de actividades como la administración general, planeación, finanzas, contabilidad, administración de aspectos legales, asuntos del gobierno y la administración de la calidad, que brindan soporte a toda la cadena de valor.

Al conocer las actividades que intervienen en la creación de valor, es posible identificar aquellas que pueden generar una ventaja competitiva, y con ello diseñar e implementar estrategias que puedan adoptar las empresas, a pesar de pertenecer a distintos giros industriales.

A través de la cadena de valor se identifican las actividades que crean valor para los clientes y las actividades de apoyo involucradas, además el reflejar los costos que genera el cumplimiento de las actividades que forman parte del proceso productivo, se convierte en un elemento fundamental para determinar la estructura de costos de una organización. Al representar costos y limitar activos, su análisis y consideración brindan las pautas para mejorar la eficiencia tecno-económica de una empresa, un grupo de empresas o un determinado sector industrial (Quintero y Carreño, 2005).

\section{METODOLOGÍA}

La presente investigación es de tipo cualitativa con un alcance descriptivo y explicativo, debido a que se identifican y presentan las principales características de la cadena de valor del sector cocotero de la región Costa Grande del Estado de Guerrero, retomando a Porter (2015), Kumar y Kapoor (2010).

La información se encuentra respaldada principalmente mediante fuentes de información primaria, tal como la aplicación de entrevistas estructuradas a las empresas que procesan los diferentes derivados del coco, entre- vistas semiestructuradas a Instituciones de Educación Superior y la observación directa.

Respecto a las entrevistas semiestructuradas dirigidas a las empresas que transforman los diferentes derivados del coco, se aplicó un instrumento con base en el Instituto Interamericano de Cooperación para la Agricultura (IICA,2016), así como del Consejo Nacional de Ciencia y Tecnología y el Instituto Nacional de Estadística y Geografía (CONACYT \& INEGI, 2014), este último con el objetivo conocer las características de los avances en materia de investigación, desarrollo tecnológico e innovación desarrollados por las empresas. La población de estudio se conforma por un total de 15 empresas ubicadas en la región Costa Grande, de la cuales por cuestiones de seguridad se realizó un tipo de muestreo no probabilístico por conveniencia, seleccionando un total de 11 organizaciones de diferentes municipios que fueron nominadas por el Consejo Estatal del Cocotero O.P.D. al ser las más representativas. Las entrevistas fueron aplicadas en el periodo abrilmayo de 2018. La estructura del cuestionario dirigido a empresas que procesan derivados de coco es la siguiente (Tabla 1).

En el caso de las entrevistas semi-estructuradas realizadas a Instituciones de Educación Superior ubicadas en la región Costa Grande, cuya finalidad fue conocer los proyectos que se han desarrollado en vinculación con el sector cocotero se consideraron los siguientes aspectos (Tabla 2).

\section{RESULTADOS Y DISCUSIÓN}

De acuerdo a la metodología aplicada, se identificaron las actividades que realizan las empresas que procesan los diferentes derivados del coco, clasificándolas en actividades primarias y actividades de apoyo, las cuales se describirán a continuación y se ilustran en la figura 1.

Tabla 1. Cuestionario dirigido a empresas que procesan derivados de coco. Fuente: Elaboración propia.

\begin{tabular}{clc}
\hline Instrumento 01. Cuestionario dirigido a empresas que procesan derivados de coco \\
\hline Sección & \multicolumn{1}{c}{ Tema de la sección } & Ítems \\
I & Caracterización del productor & 14 \\
II & Caracterización de la planta & 7 \\
III & Caracterización de la producción & 5 \\
IV & Caracterización de la & 6 \\
V & comercialización & 1 \\
VI & Costos de producción \\
VII & Ingresos anuales \\
I & Estado de resultados & 1 \\
II & Instrumento 02. Gestión de la Calidad \\
\hline Instrumento 03. Aspectos Organizativos y Socio-Empresariales \\
\hline \multicolumn{4}{c}{ Instrumento 05. Investigación y Desarrollo Tecnológico. } \\
\hline
\end{tabular}


Tabla 2. Entrevista semi-estructurada dirigida a Instituciones de Educación Superior. Fuente: Elaboración propia.

\begin{tabular}{|c|c|c|}
\hline \multicolumn{3}{|r|}{ Instrumento 02. Cuestionario dirigido a IES } \\
\hline Sección & Tema de la sección & Contenido \\
\hline I & Datos Generales & $\begin{array}{l}\text { Nombre } \\
\text { Grado Académico }\end{array}$ \\
\hline II & $\begin{array}{l}\text { Datos de la } \\
\text { Institución }\end{array}$ & $\begin{array}{l}\text { Años de operación de la institución } \\
\text { Área de conocimiento. } \\
\text { Programas de estudio ofertados. } \\
\text { Proyectos de investigación realizados. } \\
\text { Áreas de generación y aplicación de conocimientos. } \\
\text { Fortalezas y debilidades de los proyectos de investigación } \\
\text { Porcentaje del programa de estudio destinado a la aplicación de conocimiento. } \\
\text { Convenios con organizaciones gubernamentales o empresariales. } \\
\text { Conocimiento de las problemáticas. } \\
\text { Proyectos de investigación dirigidos al sector agroalimentario o agroindustrial. }\end{array}$ \\
\hline III & $\begin{array}{l}\text { Aspectos de } \\
\text { vinculación }\end{array}$ & $\begin{array}{l}\text { Vinculación como elemento clave para el desarrollo de los sectores regionales. } \\
\text { Capacidad de vinculación con el sector productivo. } \\
\text { Factores que limitan la cooperación entre IES, empresas y organizaciones } \\
\text { gubernamentales. } \\
\text { Factores que dificultan la transferencia de conocimiento. } \\
\text { Disponibilidad para la cooperación con el sector productivo. }\end{array}$ \\
\hline
\end{tabular}

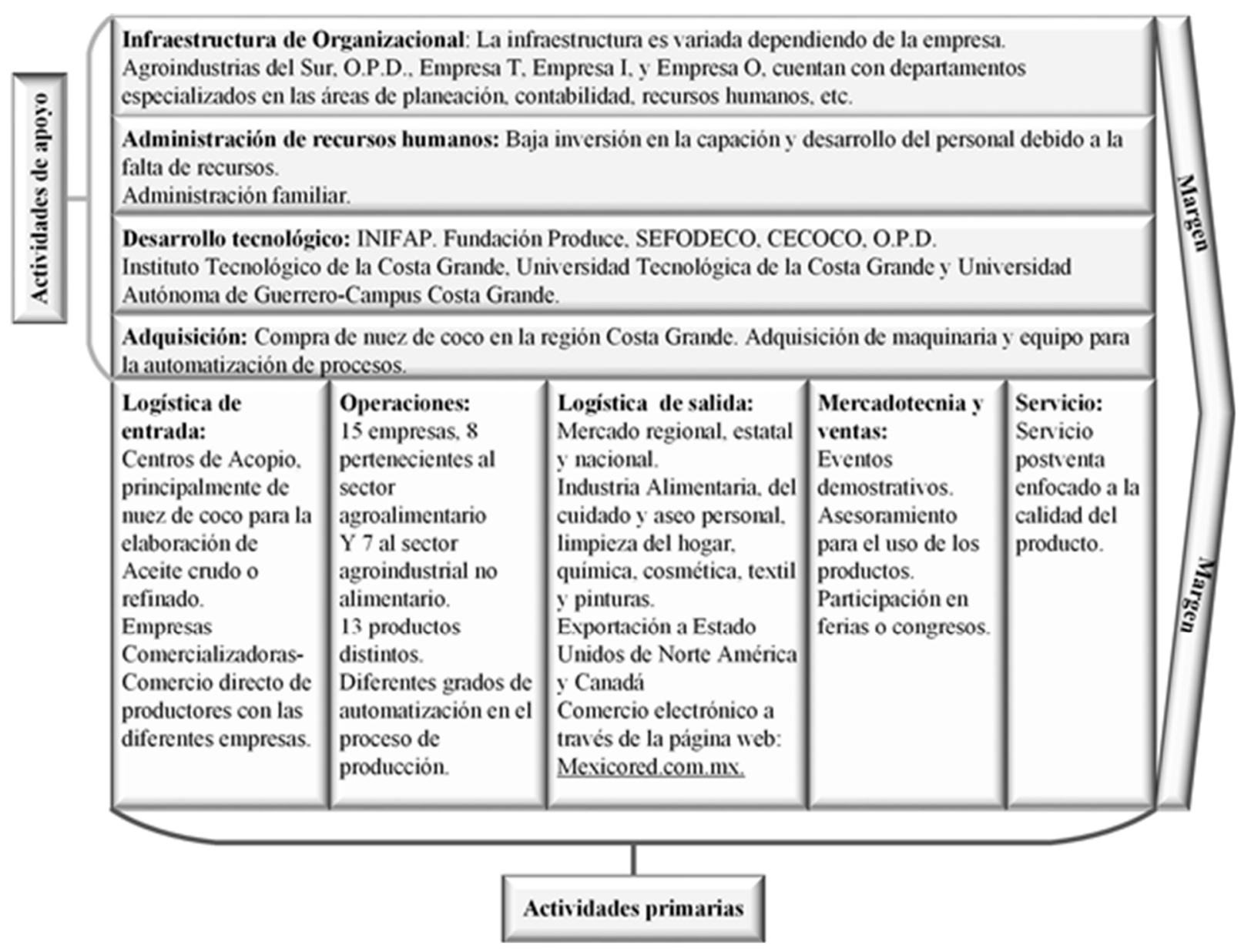

Figura 1. Cadena de Valor del Sector Cocotero en la Costa Grande de Guerrero. Fuente: Elaboración propia. 


\section{Actividades primarias}

Logística de entrada. En la región, existen diversos centros de acopio en su mayoría destinados a la elaboración de aceite de coco, entre los que se encuentra la Unión Estatal de 24 Centros de Acopio de Copra del Estado de Guerrero A.C. Además, se cuenta con empresas comercializadoras que se encargan de transportar el coco desde los huertos a las empresas procesadoras de los diferentes derivados de coco de acuerdo con la tabla 3.

Tabla 3. Comercializadoras de coco de la región Costa Grande del estado de Guerrero, 2018. Fuente: Elaboración propia.

\begin{tabular}{lll}
\hline Empresa & Producto & Mercado \\
\hline Comercializadora 1 & Coco jimado verde & Estatal \\
Comercializadora 2 & Coco jimado verde & Exportación EE. UU. \\
Comercializadora 3 & Copra & Estatal \\
\hline
\end{tabular}

El proceso de recepción de su principal materia prima en algunas empresas consiste en conocer el peso de los camiones de carga al momento de su llegada y al finalizar la descarga de producto para calcular las toneladas de transporta el vehículo. Además, se toma una muestra de copra para evaluar su acidez, y con base en los resultados se premia o sanciona al proveedor. Lo anterior, representa una de las etapas más importantes para la elaboración del aceite de coco crudo, debido a que las condiciones de la copra determinan el color, la calidad y el precio que pueda obtener la empresa por este. Las empresas que elaboran aceite crudo en el municipio de Atoyac de Álvarez y Agroindustrias del Sur, O.P.D. ubicada en San Jerónimo de Juárez se abastece directamente de productores primarios; la empresa más grande en Atoyac de Álvarez cuenta con un con un padrón de 2,800 productores en comercio directo. A pesar de ello, una de las principales problemáticas señaladas por las empresas es la falta de mecanismos que garanticen el abastecimiento de su principal materia prima y que permita el aprovechamiento de la capacidad instalada de cada una de estas.

Para la venta de coco jimado tanto para exportación como al interior de país, el acopio se realiza a través de la compra a productores que cuentan con la capacidad de trasladar el coco a las plantas donde se destopa, selecciona y empaqueta. La materia prima también es transportada por intermediarios que compran el coco a los productores primarios. Por otro lado, para la producción de sustrato de coco y fertilizante orgánico, se requiere de la cáscara fibrosa (bonote de coco), el cual se recolecta por las empresas en los patios peladeros de los productores y otras empresas que no requieren de este derivado del coco en la elaboración de sus productos. O bien, es transportado por los vendedores a las plantas productoras. En el caso particular de la Empresa O, ubicada en Técpan de Galeana, dedicada a la elaboración de biofertilizante, inoculantes y sustratos orgánicos, se requiere de desechos industriales como el aserrín, así como lodo de caña, y minerales provenientes de San Luis Potosí. Además, se realiza un intercambio con los productores de encino y mango para la recolección de las hojas residuos de sus cultivos.

Operaciones. Se han identificado un total de quince empresas, de las cuales 8 elaboran productos para el sector agroalimentario, y 7 tienen productos destinados a la agroindustria no alimentaria, quienes se encargan de elaborar 13 productos distintos: aceite de coco extra virgen, aceite de coco crudo y refinado, agua de coco, coco deshidratado, coco jimado, dulces de coco, crema, empanizador, sustrato de fibra de coco, artesanías, carbón activado y pasta de coco; la elaboración de los productos se realiza mediante procesos de producción artesanal e industrial. Las empresas presentan diferentes grados de automatización en sus procesos. Se encuentra que las empresas con más de 10 años en funcionamiento son las que han logrado transitar a un proceso de producción industrial.

La empresa I, encargada de la elaboración de aceite de coco crudo y refinado, ácidos grasos de aceite de coco y pasta de coco; con 60 años de experiencia cuentan con un proceso de producción automatizado y dispone de la única planta de refinación en el estado de Guerrero, los datos de operación se muestran en la tabla 4.

Tabla 4. Datos de Operación: Empresa I. Fuente: Elaboración propia de acuerdo con ICOSA (2018).

\begin{tabular}{|c|c|}
\hline Datos de operación & Capacidad instalada \\
\hline 1500 toneladas/mes de copra. & $\begin{array}{l}40 \text { toneladas/día de aceite de } \\
\text { coco extraído. }\end{array}$ \\
\hline $\begin{array}{l}750 \text { toneladas/mes de Aceite de c } \\
\text { coco extraído }\end{array}$ & $\begin{array}{l}100 \text { toneladas/día de aceite de } \\
\text { coco refinado (RBD). }\end{array}$ \\
\hline $\begin{array}{l}650 \text { toneladas/mes de pasta de } \\
\text { coco. }\end{array}$ & $\begin{array}{l}700 \text { de inventario de materia } \\
\text { prima } \\
1600 \text { toneladas de inventario de } \\
\text { producto terminado. }\end{array}$ \\
\hline
\end{tabular}

Otra empresa a destacar es Agroindustrias del Sur, O.P.D., este organismo público fue creado con la finalidad de impulsar el sector cocotero en la región Costa Grande, actualmente se enfoca la transformación y comercialización de aceite de coco crudo y pasta de coco (Tabla 5). Esta empresa ha optado por aumentar el número de máquinas extractoras de aceite Expeller para lograr producir continuamente y no interrumpir el proceso durante el mantenimiento de la maquinaria.

Tabla 5. Datos de operación Agroindustrias del Sur, O.P.D. Fuente: Elaboración propia.

\begin{tabular}{ll}
\hline Datos de operación & Capacidad instalada \\
\hline 200 toneladas/mes de copra. & 30 toneladas/día de aceite \\
36 toneladas/mes de pasta de & \\
coco. & \\
\hline
\end{tabular}


Por otro lado, la empresa C, ubicada en la localidad de Pantla, municipio de Zihuatanejo de Azueta y dedicada a la elaboración de agua de coco embotellada, emplea procesos de producción más rudimentarios, en los cuales la pasterización y embotellado se realizan si utilizar ningún tipo de maquinaria. Sin embargo, la empresa tiene la capacidad de producir 48,000 botellas mensuales y su producto tiene presencia en 15 estados diferentes. La presentación de venta de los principales productos elaborados por las empresas que procesan los diferentes derivados de coco en la región Costa Grande del estado de Guerrero se describen en la tabla 6.

En cuanto a la presentación más del $60 \%$ de las empresas señalaron venderlos envasados o empaquetados dependiendo producto (véase Figura 2).

Tabla 6. Principales productos elaborados en la Costa Grande de Guerrero Fuente: Elaboración propia.

\begin{tabular}{ll}
\hline Producto & Presentación \\
\hline & Pacas fibra de coco (200 y 3,300 litros) \\
& Biofertilizante sólido genérico/ Cocoplus (50 kg) \\
& Sustrato de crecimiento Cocogrom $(40 \mathrm{~kg})$ \\
& Sustrato de germinación/ Cocoblom $(20 \mathrm{~kg})$ \\
Sustrato de coco y fertilizante orgánico & Sustrato de crecimiento para frutillas/ Cocoberry (50 y $100 \mathrm{litros})$ \\
& Repelente biológico de plagas y enfermedades/ Oberia (50 kg) \\
& Coco jimado blanco \\
Coco jimado & Coco jimado canelo \\
& Coco jimado combrote \\
Agua de coco & Coco jumado blanco pintado \\
Aceite de coco crudo y refinado & Embotellada (500 milititros) \\
Aceite de coco extra virgen & Pipas (35-50 toneladas) \\
\hline
\end{tabular}

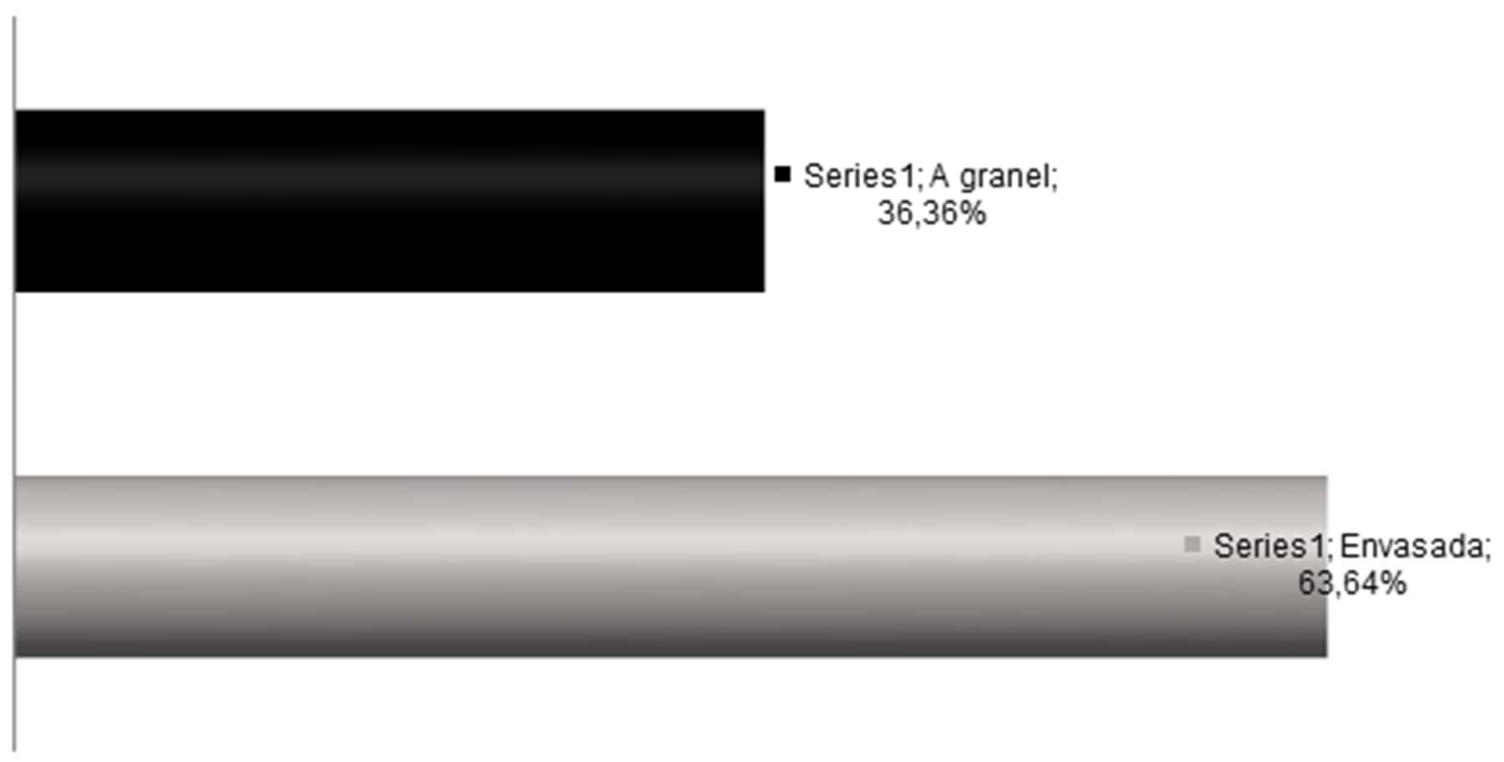

Figura 2. Presentación de venta de los productos elaborados en la Costa Grande de Guerrero. Fuente: Elaboración propia. 
Logística externa. El destino del cultivo de la nuez de coco en la región Costa Grande (Tabla 7) es el abastecimiento de los insumos que requieren las empresas del estado y una gran parte se destina a cubrir la demanda nacional; estados como Colima compran agua de coco para envasarla. Así mismo, las empresas de la región venden sus productos a nivel regional, estatal y nacional, la Empresa I atiende industrias nacionales como la alimentaria, del cuidado y aseo personal, limpieza del hogar, química, cosmética, textil y pinturas. Algunas comercializan coco jimado a Estados Unidos de Norte América y Canadá. Los productores de coco y las empresas también comercializan sus productos a través de la página web http://mexicored.com.mx/, este medio pone a disposición coco fresco, y diferentes productos derivados de este y los clientes interesados pueden contactar a los distintos proveedores. La Empresa $\mathrm{O}$ envía sus productos a Cuernavaca, Morelos y a la zona del bajío mexicano.

Mercadotecnia y ventas. Algunas de las actividades que realiza la Empresa $O$ para inducir a los consumidores a usar sus productos es la elaboración de eventos demostrativos a fin de conocer los beneficios de emplear los sustratos y fertilizantes que elaboran, además se brinda asesoramiento a los usuarios para adecuarlos a las necesidades de sus cultivos. Ante la falta de recursos para invertir en una estrategia de marketing a gran escala, varias empresas han optado por participar en ferias o congresos, donde exponen sus bienes.

Servicio. Las empresas más consolidadas son las que realizan servicios postventa, este servicio se enfoca principalmente en la calidad de los productos, en el caso de la Empresa $O$, se realiza un seguimiento del funcionamiento de sus fertilizantes y sustratos en diferentes cultivos, esta actividad sirve para retroalimentar los conocimientos de sus productos.

\section{Actividades secundarias o de apoyo}

Infraestructura organizacional. Las actividades de apoyo con relación a la planeación, contabilidad de las empresas, recursos humanos, varían de una empresa a otra; se identifican empresas como Agroindustrias del sur O.P.D., Empresa T, Empresa I y Empresa O, que cuentan con departamentos especializados en realizar dichas actividades.
Por otro lado, existen empresas en el sector que no cuentan con personal capacitado para la llevar a cabo la administración de las actividades de apoyo.

Administración de recursos humanos. En el sector son pocas las empresas que realizan actividades relacionadas con la búsqueda de personal y la contratación de este o actividades relacionadas con la capacitación y desarrollo del personal; este es el caso de las pequeñas empresas que son administradas por familias. Debido al grado de desarrollo en el que encuentran algunas organizaciones, destinar recursos a cubrir dichas actividades no está a su alcance. De acuerdo con su página web, la Empresa I realiza programas de responsabilidad social, que abarcan el desarrollo de capital humano y buscan tener un compromiso ético con sus socios y proveedores de materia prima (ICOSA, 2018).

Desarrollo tecnológico. Diversas instituciones gubernamentales colaboran con los productores de coco y las empresas que conforman el sector para el desarrollo investigaciones que aumenten la competitividad del sector y permitan el aprovechamiento integral de los diferentes derivados del coco. Entre los que se encuentra, la Secretaría de Fomento y Desarrollo Económico (SEFODECO), el Instituto Nacional de Investigación Forestal y Agrícola y Pecuaria (INIFAP), el Consejo Estatal del Cocotero O.P.D. (CECOCO) y Fundación Produce. De igual manera, se ha identificado la colaboración con Instituciones de Educación Superior (Tabla 8), principalmente las que se encuentran ubicadas en la región Costa Grande del estado de Guerrero.

Adquisición. Las empresas que conforman el sector se abastecen de nuez de coco en la región; esta es su principal materia prima, durante la recepción de esta materia prima se realiza un proceso de selección para conocer el destino que tendrá cada nuez de coco, esto depende del grado de maduración de este al momento de la cosecha. La mayoría de las empresas que procesan los derivados del coco compran por gruesa que equivale a 72 pares de nuez de coco. La compra de maquinaria y equipo la realizan a empresas nacionales e internacionales, y tiene como objetivo la automatización de los procesos de producción.

Tabla 7. Destino de los principales productos elaborados en la Costa Grande de Guerrero. Fuente: Elaboración propia.

\begin{tabular}{ll}
\multicolumn{1}{c}{ Producto } & \multicolumn{1}{c}{ Destino } \\
\hline \multirow{2}{*}{$\begin{array}{l}\text { Sustrato de coco y fertilizante orgánico } \\
\text { Coco jimado }\end{array}$} & Guerrero, Morelos, Durango, Veracruz, Chiapas, \\
Aceite de coco extra virgen & Colima, EE.UU, Canadá. \\
& Guerrero, Estado de México y Baja California. \\
Agua de coco & Michoacán, Querétaro, San Luis Potosí, Jalisco, \\
Aceite de coco crudo y refinado & Estado de México y Zacatecas. \\
\hline
\end{tabular}


Tabla 8. IES que colaboran con el sector cocotero en la Costa Grande de Guerrero. Fuente: Elaboración propia.

\begin{tabular}{ll}
\hline \multicolumn{1}{c}{ Instituciones de Educación Superior } & \multicolumn{1}{c}{ Ubicación } \\
\hline $\begin{array}{l}\text { Universidad Tecnológica de la Costa Grande del Estado } \\
\text { de Guerrero }\end{array}$ & $\begin{array}{l}\text { Carretera Nacional Acapulco Zihuatanejo } \\
\text { Kilómetro 201, Ejido el Cocotero, 40830 Petatlán, } \\
\text { Gro. }\end{array}$ \\
$\begin{array}{l}\text { Universidad Autónoma de Guerrero-Campus Costa } \\
\text { Grande }\end{array}$ & $\begin{array}{l}\text { Carretera Nacional Acapulco-Zihuatanejo, } \\
\text { Acapulco - Zihuatanejo 105, Tecpán de Galeana, } \\
40900 \text { Gro. } \\
\text { Instituto Tecnológico de la Costa Grande }\end{array}$ \\
$\begin{array}{l}\text { Manzana 30, Lote 1, Col. El Limón, C.P. 40880, } \\
\text { Zihuatanejo, Guerrero. }\end{array}$
\end{tabular}

\section{DISCUSIÓN}

A partir del análisis de cada una de las subactividades que integran las dos categorías mencionadas, se identificó que las empresas, independientemente de los productos que elaboran presentan similitud en la logística de entrada, ya que la recepción de materia prima se realiza por medio de tres mecanismos: centros de acopio, compra directa a los productores e intermediarios. Sin embargo, debido a la demanda de coco por parte de las empresas de otros estados, principalmente colima, se ha señalado la falta de abastecimiento de la materia prima, situación por la cual, no existe un aprovechamiento de la capacidad instalada con que cuentan las empresas de la región, como una de las principales problemáticas en relación las subactividades antes mencionada. Mejorar los nexos de la cadena de valor de la empresa con la cadena de valor de los proveedores representan una oportunidad para mejorar su ventaja competitiva. Al mismo tiempo, esta situación beneficiaría a los productores involucrados al garantizarles un mercado al cual vender su cosecha. Al mejorar su relación con los proveedores las empresas adoptarían un requisito indispensable para la competitividad, que es el cumplimiento de la Norma ISO 9001, la cual implica que los proveedores sean seleccionados, evaluados y reevaluados.

Para Sarache-Castro et al. (2009), la función del aprovisionamiento exige procesos logísticos más eficientes que representen una ventaja competitiva, fijando sus metas en función del costo, calidad, la disponibilidad, plazos de entrega y servicios. Considerando que la calidad de la materia prima es un aspecto importante para determinar mejores atributos en los productos derivados del coco, el esfuerzo de las empresas de la región por mejorar las condiciones de esta, y garantizar su abastecimiento, es una estrategia que pueden adoptar en conjunto. Gómez et al. (2008), señalan que la etapa de aprovisionamiento la vinculación con la etapa de producción debe ser estrecha, de tal manera que respondan a las necesidades de las áreas productivas garantizando cubrir las necesidades del producto final y buscando reducir los costos de inventarios de materias primas.

Caso contrario a la logística de entrada, donde existen similitudes en las empresas bajo estudio, es la logística de operación, en la cual, se distinguen procesos de la elaboración de productos completamente distintos, esto se debe al nivel de desarrollo de las organizaciones y a los años que llevan dentro de la industria, ya que aquellas empresas con más de 10 años de participación en el sector son las que han logrado procesos de producción más sofisticados.

A través del estudio de la cadena de valor del sector cocotero en la región Costa Grande, aplicando el modelo de cadena de valor y ventaja competitiva se identificaron las siguientes características:

El acopio de materia prima se realiza a través de los centros de acopio, el comercio directo con los productores y principalmente a través de intermediarios. Una de las principales problemáticas señaladas por las empresas en esta actividad es la falta de mecanismos que garanticen el abastecimiento de la materia prima y que permita el aprovechamiento de la capacidad instalada de cada una de estas. Esto se puede explicar debido a la gran participación de intermediarios estatales y nacionales dentro de la cadena de valor; dinámica que a su vez afecta a los productores primarios, ya que al mantener bajos márgenes de ganancias su capacidad de aumentar la inversión en el desempeño de actividades, como la comercialización de sus cosechas, es limitada.

\section{CONCLUSIONES}

Ante las diferencias en la cadena de valor de las empresas que conforman el sector cocotero en la región Costa Grande del estado de Guerrero, resulta compleja la definición de estrategias que puedan ser adoptadas por cada una de ellas a fin aumentar su competitividad y tener una mayor participación tanto a nivel estatal como nacional. Sin embargo, las debilidades o deficiencias pueden verse fortalecidas beneficiando al sector cocotero en general, lo que permite la integración de los productores primarios.

Las empresas que cuentan con mayor experiencia en el mercado son las que han logrado automatizar sus procesos de producción; por otro lado el desarrollo tecnológico ha sido posible por medio de la colaboración con instituciones gubernamentales e Instituciones de Educación Superior.

De acuerdo con la Agenda de Innovación de Guerrero (2015), en el período 2009-2013, solo cinco firmas 
guerrerenses han obtenido más de un proyecto en el Programa de Estímulo a la Innovación, lo que refleja la falta de continuidad de las acciones privadas en el ámbito de la innovación. Por ello, la participación y el aprovechamiento de la estructura científico-tecnológica con la que cuenta el estado de Guerrero se convierte en una alternativa para aumentar la capacidad de las empresas ubicadas en la región Costa Grande y, por ende, mejorar su competitividad.

\section{AGRADECIMIENTOS}

Al Consejo Nacional de Ciencia y Tecnología (CONACyT) por el apoyo brindado para la realización de la presente investigación a través de la beca 470351. Asimismo, a la Universidad Autónoma de Guerrero por el apoyo a través del proyecto "Gestión e impulso de la innovación para la competitividad de las cadenas agroalimentarias" en el marco de la Convocatoria para Financiamiento de Proyectos Semilla 2018. Al Consejo Estatal del Cocotero, O.P.D. quienes brindaron las facilidades que hicieron posible el desarrollo de la investigación.

\section{REFERENCIAS}

Acosta, M.; D. Coronado \& C. Romero, C. 2015. Linking public support, R\&D, innovation and productivity: New evidence from the Spanish food industry. Reino Unido. Food Policy. 57: 50-61.

Arredondo, V. 1990. La educación superior y su relación con el sector productivo: problemas de formación de recursos humanos para el desarrollo tecnológico y alternativas de solución. México. Revista de la Educación Superior. 76.

CECOCO. 2016. Recursos Estatales 2016. Justificación del gasto corriente. Técpan de Galeana, Gro. Recuperado de http://i.guerrero.gob.mx/uploads/2016/03/2PRO-PROGRAMAS-Y-PROYECTOS-DE-

INVERSI\%C3\%93N-32.pdf Último acceso: junio de 2019.

CONACYT \& INEGI. 2014. Encuesta Sobre Investiga- ción y Desarrollo Tecnológico. Recuperado de: http://www.beta.inegi.org.mx/contenidos/proyectos/ence stablecimien-

tos/especiales/esidet/2014/doc/cuestionario.pdf. Último acceso: octubre de 2020.

Gómez, H.; R. Cáceres \& N. Mancilla. 2008. Las Pymes: costos en la cadena de abastecimiento. Colombia. Revista Escuela de Administración de Negocios. 63: 522.

IICA. 2016. Metodología para la Identificación Territorial de Innovaciones Tecnológicas para mejorar la Competitividad y Sustentabilidad de los Agronegocios. Panamá. IICA. 50 pp.

Kumar, N. \& S. Kapoor. 2010. Value Chain Analysis of Coconut in Orissa. Suecia. Agricultural Economics Research Review. 23:411-418.

ICOSA. 2018. Recuperado de: http://icosa.mx/\#about

Malerba, F. 2002. Sectoral systems of innovation and production. Países Bajos. Research Policy. 31: 247264.

Organización de las Naciones Unidas para la Alimentación y la Agricultura. 2017. FAOSTAT. Recuperado

de:

http://www.fao.org/faostat/es/\#data/QC/visualize Ultimo acceso: octubre 2020.

Porter, M. 2015. Ventaja competitiva: creación y sostenimiento de un desempeño superior. México. Grupo Editorial Patria.

Quintero, J., \& Carreño, J. 2005. La cadena de valor: Una herramienta del pensamiento estratégico. Venezuela. Telos Revista de Estudios Interdisciplinarios en Ciencias Sociales. 8(3): 377-389.

Sagheer, S., \& Yadav, S. 2009. Developing a conceptual framework for assessing competitiveness of India's agrifood chain. Reino Unido. International Journal of Emerging Markets. 2 (4): 137-159.

Sarache-Castro, A.; Castrillón-Gómez, D. \& OrtizFranco, F. 2009. Selección de proveedores: una aproximación al estado del arte. Colombia. Cuadernos de Administración. 22(38): 145-167.

SIAP. 2018. Anuario Estadístico de la Producción Agrícola. Obtenido de http://infosiap.siap.gob.mx/aagricola_siap_gb/icultivo/in dex.jsp Ultimo acceso: octubre 2020. 